
cultures

Les cahiers de l'Acedle

\title{
Enseignement de la littérature dans les lycées marocains : de la centration sur les genres littéraires classiques à l'apprentissage du goût de la lecture
}

Mina Sadiqui

\section{(2) OpenEdition \\ Journals}

Édition électronique

URL : https://journals.openedition.org/rdlc/7819

DOI : $10.4000 /$ rdlc.7819

ISSN : 1958-5772

Éditeur

ACEDLE

Référence électronique

Mina Sadiqui, «Enseignement de la littérature dans les lycées marocains : de la centration sur les genres littéraires classiques à l'apprentissage du goût de la lecture », Recherches en didactique des langues et des cultures [En ligne], 17-2 | 2020, mis en ligne le 27 avril 2020, consulté le 17 octobre 2022 URL : http://journals.openedition.org/rdlc/7819; DOI : https://doi.org/10.4000/rdlc.7819

Ce document a été généré automatiquement le 17 octobre 2022.

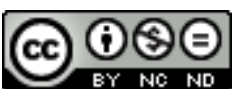

Creative Commons - Attribution - Pas d'Utilisation Commerciale - Pas de Modification 4.0 International - CC BY-NC-ND 4.0

https://creativecommons.org/licenses/by-nc-nd/4.0/ 


\title{
Enseignement de la littérature dans les lycées marocains : de la centration sur les genres littéraires classiques à l'apprentissage du goût de la lecture
}

\author{
Mina Sadiqui
}

\section{Introduction}

1 L'enseignement/apprentissage du $\mathrm{FLE}^{1}$ au lycée au Maroc repose depuis $2002^{2}$ sur l'approche par compétence et se base sur un objet/support spécifique: l'œuvre intégrale. L'introduction de la littérature en classe de langue doit inscrire la discipline dans une triple perspective : linguistique, méthodologique et culturelle (Ministère De l'Education Nationale Marocain (MEN), 2007a).

2 Dans le dernier texte officiel intitulé «Les orientations pédagogiques générales pour l'enseignement du français dans le cycle secondaire qualifiant", édité par le Ministère National d'Education Nationale, toujours en vigueur (2007a), l'entrée par les genres est préconisée pour enseigner et apprendre le français au lycée. On retrouve cette conception de la notion chez Todorov $(1970: 12)$ qui considère que « les genres sont ces relais par lesquels l'œuvre littéraire se met en rapport avec l'univers de la littérature ».

3 Les genres littéraires sont des catégories de classement des textes littéraires, des conventions discursives telles que les dénombre Jean-Marie Schaeffer (1989). La notion peut être « un pivot autour duquel bâtir un certain nombre de compétences » (Canvat, 1999 : 7). Nous pensons toutefois qu'il serait pertinent de repenser son statut et ses diverses fonctions dans un dispositif d'enseignement/apprentissage dans une classe de langue, de façon à transformer la notion en véritable outil de lecture et d'écriture, optimisant ainsi ses divers usages et ouvrant la discipline à d'autres corpus. 
Comment donc aborder les genres littéraires à approcher dans une classe FLE de sorte que l'approche puisse contribuer à la construction des compétences de lecture et d'écriture de notre apprenant? Comment l'approcher tout en repensant les contenus et les démarches mises en place et donc en revisitant les repères de la discipline «français » au lycée au Maroc?

5 Nous essayerons dans cette contribution de donner quelques pistes d'exploitation didactique, en nous inspirant, d'une part, des dernières avancées en didactique de la littérature grâce aux théories de la réception (Eco, 1987 ; Iser, 1985) qui valorisent le sujet lecteur, et d'autre part, des nouvelles théories de l'apprentissage tels le cognitivisme (Piaget, 1975) ou encore le socioconstructivisme (Vygotsky, 1934) qui placent l'apprenant au centre de la situation d'enseignement /apprentissage.

\section{« Genres littéraires » et discipline « français » au lycée}

\section{Statut et fonction du genre du discours littéraire dans le texte officiel marocain}

6 Le dernier programme officiel (MEN, 2007), toujours en vigueur, assigne une place spécifique à la notion de genre littéraire. Nous avions précisé auparavant que le discours officiel préconise le texte littéraire comme le principal support d'enseignement/apprentissage en classe de langue au lycée.

7 La notion de genre figure dans le référentiel de compétences que tout apprenant doit construire à l'issue du cycle secondaire qualifiant (lycée). Ainsi, elle y est bien ciblée comme compétence à construire. Il est certes précisé qu'en fin du cycle tout apprenant est censé être capable de « reconnaître les différents genres d'écrits » (MEN, $2007: 4$ ), mais, et comme nous le démontrerons c'est l'écrit littéraire qui est privilégié.

En outre, la notion justifie le choix des œuvres proposées. C'est dans ce sens qu'on y souligne que les œuvres retenues, avec une nette prédominance de genre romanesque classique $^{3}$, ont pour finalité d'« initier les élèves aux principaux genres et mouvements littéraires » (MEN, $2007:$ 12).

9 La notion joue un rôle essentiel dans la conception des dispositifs didactiques vu que le module, dispositif fondamental de mise en œuvre du projet pédagogique annuel, «s'articule principalement autour d'une œuvre intégrale» (MEN, 2007:6) et que comme nous l'avions précisé, la notion du genre est déterminante dans le choix de cette dernière dans les programmes préconisés.

10 La mise en œuvre des dispositifs pédagogiques de certaines activités de classe prônées, l'activité de lecture en l'occurrence, met en exergue une conception particulière de la notion. En effet, les approches de lecture recommandées (MEN, 2007:6) semblent concevoir le genre littéraire comme support et objet d'enseignement/apprentissage en lecture et en écriture, un objet de savoir ayant pour finalité la transmission d'une culture.

11 La notion de genre est enfin fondamentale dans la conception des situations évaluatives, l'examen certificatif en l'occurrence, où la contextualisation et la détermination générique sont des questions récurrentes dans toute étude des textes proposés à l'apprenant : 
"Supports d'évaluation:

Ils sont à retenir en fonction des objectifs visés. On aura recours à l'un des types de

supports suivants :

- un extrait d'une œuvre intégrale,

- deux extraits d'une même œuvre,

- des extraits appartenant à des genres différents,

- un extrait d'une œuvre en dehors du programme, mais appartenant à l'un des

genres littéraires étudiés,

- un texte didactique (définition de la tragédie, de l'autobiographie, du réalisme...)

et un extrait d'une œuvre illustrant la définition, etc.,

- un texte poétique, etc. (MEN, $2007: 12)$.

Dans le cadre de référence de l'examen normalisé régional pour l'obtention du baccalauréat, la reconnaissance de la notion du genre est bien présente. Ce texte qui énonce les paramètres qui serviront de fondement à l'évaluation des acquis des élèves de la première année du cycle du baccalauréat, toutes sections confondues, constitue un document de référence permettant de cibler les éléments essentiels et représentatifs à évaluer. Parmi les trois capacités retenues à valider dans le cadre de cette évaluation effective, celle qui est appelée «contextualiser ", figure en première place.

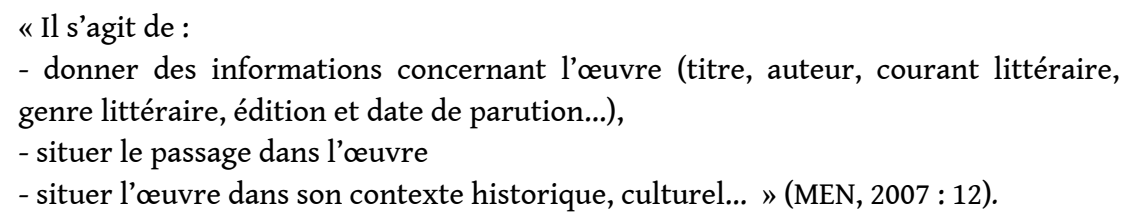

\section{Statut et fonction du genre littéraire dans les « progressions »}

Les «progressions" sont des documents recommandés par le ministère et qui complètent le contenu proposé par le texte officiel. Elles développent des propositions pratiques de dispositifs d'enseignement /apprentissage à partir des corpus préconisés. Elles explicitent, à travers les activités proposées, les démarches conceptualisées dans le texte officiel. En effet, ces documents fournis aux praticiens proposent plusieurs genres considérés comme moyens pédagogiques mobilisables par l'enseignant. Dans ce sens, au cycle secondaire qualifiant, tout apprenant doit étudier une nouvelle réaliste de Maupassant, une nouvelle fantastique de Mérimée, une comédie de Molière, un roman autobiographique de Sefrioui, un roman à thèse de Victor Hugo, un roman de Balzac et un conte de Voltaire (MEN, 2007).

Les progressions proposées sont organisées par référence aux divers genres, en vue de transmettre de prime abord une facette de l'histoire (littéraire) de la littérature française.

Les activités proposées dans ces progressions se répartissent selon les genres considérés. Nous avançons ces quelques exemples à titre indicatif: à partir de la nouvelle de Maupassant, on y précise que la compétence ciblée est celle de « reconnaître un genre littéraire : la nouvelle réaliste » (MEN, 2007: 20), et l'on y propose comme activité de production écrite de: «Produire un écrit d'invention » (MEN, $2007: 20$ ).

16 Pour ce qui est du dispositif à mettre en œuvre à partir d'un texte de théâtre, le «Bourgois gentilhomme » de Molière, la compétence à construire chez l'apprenant serait d' « étudier un genre littéraire : le théâtre (la comédie) » (MEN, $2007: 22)$, et dans 
ce sens l'enseignant pourrait donner comme consigne dans le cadre d'activités de productions écrites ou orales: "Réécrire une scène ", "Jouer une scène comique " (MEN, $2007: 22$ )

17 Enfin, notre dernier exemple décrit le dispositif proposé à partir d'un autre genre spécifique, à savoir la poésie, le sonnet plus particulièrement. Reconnaître les caractéristiques formelles du sonnet est la compétence ciblée. Comme activités de classe à proposer, nous relevons : « jouer avec des rimes, jouer avec des formes » (MEN, $2007: 22)$

Que retenir de ces exemples de dispositifs proposés à partir de supports appartenant à des genres différents?

19 La notion du genre littéraire aide sur le plan didactique

à concevoir des dispositifs d'enseignement/apprentissage cohérents (Canvat, 1999). Au niveau des pratiques de classe, elle contribue à la mise en œuvre d'une progression pédagogique par des activités diverses, celles relatives à la réception et celles qui permettent de passer à des activités de production orales et éventuellement scripturales. La notion tisse des liens entre les activités de lecture et d'écriture

(Daunay, Reuter et Schneuwly, 2011).

Cette approche permet donc divers usages en classe de langue au lycée, mais la notion utilisée ne revêt dans ces prescriptions qu'un seul statut : le

genre est représenté comme un objet

de savoir ayant pour finalité la transmission d'une culture, un objet disciplinaire à apprendre nécessairement pour savoir lire et éventuellement écrire dans la langueculture cible. En conséquence, « le genre est perçu comme un prototype » (Boré, 2007 :

75). Il y a une mise en valeur de son fonctionnement textuel et de ses spécificités linguistiques qui le modéliseraient.

En outre, le genre s'affiche comme un modèle textuel et une structure générique stables, ce qui pourrait induire à une représentation figée et fermée de la littérature. Cette dernière risque de rester enfermée dans le cadre traditionnel issu de la poétique en respectant la triade, roman, théâtre, poésie.

La représentation scolaire des genres est stabilisée autour de quelques grandes catégories littéraires.

Cette conception de la notion peut réduire l'approche du texte à un " simple exercice de reconnaissance » (Descotes, $1995: 11)$. Cette démarche ne conduirait-elle pas à «l'exclusion de l'expérience subjective du sujet lecteur au profit d'une description objective du fonctionnement textuel »? (Langlade, 2002 : 17-28). Mettre en valeur les problématiques génériques risque de valoriser « une approche désincarnée de la littérature, éloignée de tout investissement personnel » ( Ibid.,

2002 : 17-28) de toute implication des apprenants, au lieu de valoriser « les expériences de lecture imprévues qu'ils sont en droit de réaliser ».

(Rouxel et Langlade, $2004: 13$ ).

Les dernières décennies ont vu se renouveler les recherches en lecture et en écriture grâce aux nouvelles théories linguistiques et aux théories de la réception. Ces avancées, comme nous allons les voir, renouvellent l'approche du texte littéraire et invitent à repenser la notion du genre littéraire en contexte scolaire. 
Le sens d'un texte n'existe que pour et par sa lecture et les opérations qu'effectue le lecteur importent autant que les contenus et les formes supposés du texte (Dufays,

Pour mieux expliciter notre propos, quelques rappels s'imposent.

\section{Pour approcher autrement les genres littéraires en classe de langue au lycée au Maroc}

\section{Genre littéraire et lecteur}

Les approches $\mathrm{du}$ texte littéraire en usage ces dernières années relèvent essentiellement des théories du texte (Eco, 1965 ; Todorov 1978 ; Barthes, 1975 ; Derrida 1967). Elles postulent que tout texte est une œuvre finie. Lire, c'est retrouver l'intention de l'auteur en faisant appel à des connaissances plus ou moins savantes sur le genre, et les divers contextes. Ces approches externes centrées sur l'approche générique sont celles qui prévalent encore dans les explications de textes dans certaines situations de réception.

Avec les théories de la lecture (Eco, 1987; Iser, 1985 ; Jouve 1993), il s'opère un net déplacement de l'objet texte vers le sujet lecteur, ce qui met en valeur l'activité de ce dernier comme composante fondamentale dans "l'actualisation» du sens de l'objet littéraire. Le sens ne provient plus seulement du texte, ni de son auteur, mais aussi du sujet lecteur. Tant qu'il n'est pas lu, tout texte demeure inachevé. Ces théories, qu'elles soient centrées sur l'effet du texte ou sur sa réception, mettent en valeur l'instance du lecteur (Eco, 1987; Dufays, 1994 ; Langlade, 2004). C'est l'acte de lire qui confère au texte son caractère littéraire (Dufays, 1994 ; Tauveron, 1999 ; Langlade, 2004). Un texte littéraire est «en premier lieu l'accomplissement d'un acte de communication interhumaine, un message émis par une personne donnée dans des circonstances et avec un but spécifique, reçu par une autre personne dans des circonstances et avec un but non moins spécifique » (Schaeffer, 1989 :80).

\section{L'apprenant au centre de la situation d'enseignement/apprentissage}

La centration sur l'apprenant est une démarche issue du mouvement constructiviste. À partir des années 1970-1980, il s'est avéré que ce n'est pas l'enseignement qui produit l'acquisition d'une langue, mais l'apprentissage par un apprenant actif. Dans l'approche constructiviste, c'est le processus d'apprentissage et non le processus d'enseignement qui est le point de départ de l'acquisition d'une langue. L'activité de classe qui découle du besoin et de l'intérêt de l'apprenant permet au dispositif didactique d'être centré sur les outils facilitant la construction de son autonomie (Tardif, 1992).

Dans cette approche, le genre du discours littéraire ne peut être conçu comme simple objet disciplinaire à transmettre en lecture et à produire en écriture. Ce sont «les pratiques langagières signifiantes, socialement reconnues qui doivent orienter l'enseignement » (Dolz et Gagnon, 1998 : 178-179).

$29 \mathrm{Au}$ niveau de la gestion pédagogique, cette nouvelle conception permet le déplacement du sens expliqué par l'enseignant aux élèves au sens produit par les élèves avec l'aide de l'enseignant. 
Enfin, l'évaluation va en conséquence faire appel à l'expérience personnelle de l'apprenant. Par ailleurs, introduire de «nouveaux » supports/genres dont les thèmes et les idées se rapprochent de leurs expériences personnelles, de leur goût, de leurs préoccupations et de leurs univers (Langlade, 1994): romans d'aventure, romans policiers, science-fiction, L'introduction de ces textes n'aiderait-elle pas à mettre en œuvre ces nouvelles approches?

\section{De l'enseignement de la littérature classique à l'apprentissage d'une pratique culturelle}

\section{Repenser « le genre littéraire » pour redéfinir les repères d'une discipline}

31 Envisager les genres en prenant en considération d'abord les expériences de lecture (ou de non-lecture) de nos élèves, en dehors des cadres strictement rhétoriques et poétiques, ne va-t-il pas remettre en question ces choix « institutionnels » et permettre d'intégrer, au moins de façon partielle en contexte scolaire, ce qui est souvent nommé «paralittérature»?

La place de la littérature classique comme seul modèle culturel n'est-elle pas à relativiser?

\section{Une nouvelle approche : la lecture impliquée}

Les théories de la lecture et de la réception peuvent aider à mettre en place « la lecture impliquée » : « impliquée, c'est-à-dire fondée sur la rencontre d'un individu en situation et d'une œuvre (...) si le professeur garde l'initiative du discours sur l'œuvre, s'il protège son monopole de la mise en question (...) les chances sont minces d'une rencontre avec les apprenants.

》(Dumortier et Lebrun, $2006: 6-7$ )

. Cette démarche suppose d'écouter d'abord les réactions « de nos apprenants/lecteurs tout en conservant leur spontanéité, leur réception de l'ouvrage » (Lara, 2010 : 206 ). Cette approche du texte littéraire se base dans une première étape sur les diverses représentations du lecteur. La didactique des sciences (Astofli, 1990) a prouvé que chaque apprenant possède des conceptions préalables « qui font partie d'un système de représentations qui a sa cohérence et ses fonctions d'explication du monde » (Perrenoud, 1999: 29) et ses représentations sont foncièrement liées à sa propre culture, à sa réalité socioculturelle.

«Le fait de susciter chez les élèves des interrogations sur le pourquoi des textes, sur la réalité et l'imaginaire auxquels ils renvoient, sur les questions auxquelles ils répondent, représente une manière de les impliquer dans cette communication particulière qu'est la littérature et contribue en partie à construire leur réception ». (Rouxel, 1996 : 198). 
un obstacle de lecture, l'élève apprend à chercher les outils pertinents, et donc formateurs, pour entrer dans le texte. La démarche prend en considération le profil spécifique de l'apprenant, en même temps que ses compétences effectives, avant de penser, si le contexte le permet, à « ouvrir la lecture littéraire » (Rouxel, 1996 : 206), qui

« renvoie à des opérations cognitives de haut niveau, qui au-delà de la compréhension permettent d'interpréter le texte et surtout de jouer avec ses différentes significations » (Tsimbidy, $2008: 13)$.

Toute introduction d'un " savoir littéraire », y compris celui sur les genres littéraires, est le résultat d'une réflexion didactique sur son statut et sa finalité par rapport au dispositif d'apprentissage à mettre en place. $C$ edispositif relève du statut à attribuer à la discipline dans un contexte donné.

La constitution marocaine

de 2011 (Article 5) précise que les apprenants doivent maitriser : « les

langues étrangères (par opposition aux langues officielles) les plus utilisées dans le monde, en tant qu'outils de communication, d'intégration et d'interaction avec la société du savoir, et d'ouverture sur les différentes cultures et sur les civilisations contemporaines ».

Les finalités de l'introduction des langues étrangères au sein du système éducatif du Royaume sont d'ordre communicatif et culturel. Ces finalités convergent d'ailleurs avec celles conférées à l'apprentissage des langues étrangères en didactique des langues/ cultures. Il est en effet démontré qu' «un apprentissage de langue n'est jamais indépendant d'un apprentissage culturel » (Porcher, 1986:44) et que la maitrise de la dimension linguistique n'est que «la surface d'un iceberg qui cache des réalités culturelles dont l'appréhension est nécessaire dans toute situation de communication » (Barthelemy, Groux \& Porcher 2011: 111). D’ailleurs, si la compétence de communication «s'est révélée un concept beaucoup plus opératoire que tous ceux qui l'avaient précédé dans la mesure où elle les englobe (...) s'agissant de l'enseignement de la civilisation, la compétence de communication s'est monnayée opératoirement en compétence culturelle » (Porcher, 1986 : 44).

Mais, le bilan à mi-parcours de la réforme $(2007$ b), le premier rapport annuel de l'éducation du Conseil supérieur de l'enseignement (2008), les résultats de Trends in International Mathematics and Sciences Studies (2003 et 2007), soulignent tous le constat de sérieuses défaillances quant à la maitrise des langues, avec une tendance au recul de certains indicateurs de performance. Le rapport du Ministère de 2008, tout en concevant la question des langues comme une problématique transversale du système, note dans ce sens que :

«L'examen de la stratégie linguistique actuelle révèle de nombreux dysfonctionnements, notamment à deux niveaux :

- au niveau des langues d'enseignement, avec un déphasage particulièrement préjudiciable entre le secondaire et le supérieur ;

- au niveau de l'enseignement des langues, avec une inadéquation évidente par rapport aux compétences linguistiques recherchées » (MEN, 2008 : 64).

Il serait donc tout à fait logique, voire urgent, de repenser les repères disciplinaires $\mathrm{du} \ll$ français ». 


\section{Pour construire une véritable compétence culturelle}

40 Dufay (2003: 68) rappelle que « la culture est à la fois le principe, la finalité et le moteur de l'apprentissage d'une langue (étrangère), qui vise directement et indirectement, la rencontre de l'Autre ». Cependant, la culture est un produit socio-historique mais aussi une pratique sociale. Et comme l'a bien souligné Galisson (1991), il y a d'une part, la culture littéraire, l'histoire de l'art, la culture humaniste qu'il nomme : " le cultivé », ou encore « la culture savante », et d'autre part « le culturel» qui renvoie au mode de vie, à la culture quotidienne partagée par un certain nombre d'individus et qu'il qualifie également de « culture partagée ». Dans les programmes préconisés par le ministère et mis en œuvre dans la classe de langue au lycée, c'est le texte littéraire qui constitue la seule et unique facette de la culture française. En assimilant l'enseignement apprentissage du FLE à un enseignement de textes littéraires français classiques. On survalorise la culture canonique. Les programmes de 2007 (MEN, 2007a) intègrent deux œuvres de littérature maghrébine d'expression française, mais tout le reste est constitué des grands classiques de la littérature française.

Intégrer cette double dimension attribuée au concept de culture n'aiderait-il pas à mieux redéfinir les repères de l'enseignement/apprentissage du FLE au lycée? En effet, «la culture comportementale pourrait non seulement réconcilier l'enseignement de la langue avec celui de la culture, mais aussi souligner le lien étroit que le cultivé noue avec le culturel » (Briet, Blondel et Collés, 1998 : 7).

Or le but de notre enseignement est bien d'amener l'élève à acquérir « le goût de la lecture", les compétences qui vont lui permettre d'intégrer dans sa vie la fréquentation régulière de la production culturelle d'expression française sous toutes ses formes.

\section{Conclusion}

Examiner l'approche par la notion de genre littéraire amène à repenser les contours de la discipline français dans tout notre système éducatif.

Proposer des situations d'enseignement/apprentissage basées sur des supports diversifiés pourrait contribuer à optimiser l'appropriation de la langue cible dans ses dimensions linguistique, culturelle.

Pour ce qui est du texte littéraire dont la valeur formative est indéniable, l'objectif étant de valoriser les postures interprétatives de nos apprenants et donc de construire des sujets lecteurs et scripteurs, n'est-il pas urgent de construire l'espace d'apprentissage autour des centres d'intérêt de l'apprenant, de ses lacunes et de ses besoins, et non simplement autour de corpus prédéterminés conçus comme un modèle clos?

L'apprentissage des archétypes de la littérature française est un objectif important si on l'envisage comme une rencontre avec les fondements d'un imaginaire culturel collectif mais le corpus de textes doit être réactualisé pour le contexte spécifique de l'enseignement/apprentissage du français auprès de la jeunesse marocaine afin de lui assurer un accès au vaste domaine de la production culturelle d'expression française.

Recherches en didactique des langues et des cultures, 17-2 | 2020 


\section{BIBLIOGRAPHIE}

Astolfi, P. (1990). Les concepts de la didactique des sciences, des outils pour lire et construire les situations d'apprentissage, Recherche \& formation, ${ }^{\circ} 8$, p. 19-31.

Briet, G., Blondel, A., Collès, L. (1998). Que voulez-vous dire ? Compétence culturelle et stratégies didactiques. De Boeck-Duculot. Bruxelles.

Boré, C. (2007). Construire et exploiter des corpus de genres scolaires. Namur : Presses universitaires de Namur.

Canvat, K. (1999). Enseigner la littérature par les genres, pour une approche théorique et didactique de la notion de genre littéraire. De Boeck. Bruxelles.

Daunay, B., Reuter, Y., Schneuwly, B. (2011). Les concepts et les méthodes en didactique du français. Presses universitaires de Namur. Namur.

Dufays, J.M. (2003). Le français langue étrangère et seconde : enseignement et apprentissage. Editions Mardaga. Liège.

Derrida, J. (1972). La dissémination. Paris : Éd. du Seuil.

Dufays, et al. (1996). Pour une lecture littéraire 2. Bilan et confrontations. Bruxelles : De Boeck \& Larcier.

Dolz J. \& Schneuwly, B. (1996). Genres et progression en expression écrite : éléments de réflexion à propos d'une expérience romande, Enjeux, 37/38, 49-75.

Dolz, J et Gagnon, R. (2008). Le genre du texte, un outil didactique pour développer le langage oral et écrit. Pratiques, 137-138, 179-198

Dumortier, J.L et Lebrun, M. (2006). Une formation littéraire malgré tout : enseigner la littérature dans les classes difficiles. Namur : Presses universitaires de Namur.

Eco, U. (1985) [1979]. Lector in fabula. Paris : Grasset.

Eco, U. (1965). L'œuvre ouverte. Paris : Éd. du Seuil

Galisson, R. (1991). De la langue à la culture par les mots. Paris : CLE International.

Jauss, H.R. (1978), Pour une esthétique de la réception. Paris : Gallimard.

Jouve, V. (1993). La lecture. Paris : Hachette.

Langlade, G. (2004). Sortir du formalisme, accueillir des lecteurs réels. Le français aujourd'hui, 145, 85-96.

Lara, C. (2010), Pour une réception communo-culturelle de la lecture : étude d'Atala de Chateaubriand. Paris : L'Harmattan.

Schaeffer, J.-M., (1989). Qu'est-ce qu'un genre littéraire ?. Paris : Seuil.

Piaget, J, (1975). L'Équilibration des structures cognitives : Problème central du développement (Études d'épistémologie génétique) Broché. Presses universitaires de France.

Perrenoud, Ph. (1999). Dix nouvelles compétences pour enseigner. Invitation au voyage. Paris : ESF.

Porcher, L. (1986). La civilisation. Paris : Clé International. 
Rouxel, A. et Langlade, G. (2004). Le Sujet lecteur - Lecture subjective et enseignement de la littérature. Rennes : Presses Universitaires de Rennes.

Rouxel, A. (1996). Enseigner la lecture littéraire. Presses Universitaires de Rennes.

Tardif, C. (1992). Pratiques pédagogiques facilitant l'autonomie de l'apprenant en français langue seconde. Cahiers franco-canadiens de l'ouest, vol. 4-1. 89-102.

Tauveron, C. (1999). Comprendre et interpréter le littéraire à l'école : du texte réticent au texte proliférant. Repères, 19, 9-38

Tsimbidy, M. (2008). Enseigner la littérature de jeunesse, Presses Univ. Du Mirail. Toulouse.

Todorov, T. (1987). La notion de littérature et autres essais. Paris : Seuil.

Vygotski, L.S. (1934/1985a), Pensée et Langage, traduction Françoise Sève, Paris : ed. sociales.

Documents officiels :

Ministère de l'Education Nationale (M.E.N). (2007a). Les orientations pédagogiques générales pour l'enseignement du français dans le cycle secondaire qualifiant.

Conseil Supérieur de l'Enseignement. (2008). Etat et Perspectives du Système d'Education et de Formation. Royaume du Maroc.

Ministère de l'Education Nationale. (2007b). Bilan à mi-parcours de l'Education pour tous 2000-2007. Rabat.

Ministère de l'Education Nationale. Département de l'éducation nationale, de l'enseignement supérieur, de la formation des cadres et de la recherche scientifique. (2005). Réforme du système éducatif: Bilan et perspectives. Rabat.

Secrétariat Général du Gouvernement. (2011). Constitution du Maroc. Royaume du Maroc.

Ministère de l'Éducation Nationale de l'Enseignement Supérieur de la Formation des Cadres et de la Recherche Scientifique. (2008). Pour un nouveau souffle de la réforme de l'Éducation-Formation. Présentation du Programme NAJAH. Royaume du Maroc.

Commission Spéciale Education Formation. (1999). Charte Nationale d'Education et de Formation. Royaume du Maroc.

\section{NOTES}

1. «Le concept de langue étrangère se construit par opposition à celui de langue maternelle et l'on peut dire dans un même temps que toute langue non maternelle est une langue étrangère. » (Cuq, $2002:$ 93).

2. En réponse aux principes de la Charte de d'Education et de Formation de 1999, une refonte des curricula commence. Soulignons également que la Constitution de 2011 précise que l'état doit veiller à « la maitrise des langues étrangères (par opposition aux langues officielles) les plus utilisées dans le monde, en tant qu'outils de communication, d'intégration et d'interaction avec la société du savoir, et d'ouverture sur les différentes cultures et sur les civilisations contemporaines » (Royaume du Maroc, 2011, article 5).

3. Parmi les neuf œuvres intégrales proposées aux élèves du lycée, six sont écrites par des auteurs du $19^{\text {àme }}$ ou du $18^{\text {ème }}$ siècle. 


\section{RÉSUMÉS}

Cette contribution revient sur les fonctions et les usages des genres littéraires tels que préconisés dans les programmes officiels pour le secondaire au Maroc. Certaines dérives sont alors soulignées et des pistes didactiques proposées. Ces dernières devraient contribuer à optimiser l'enseignement/apprentissage du FLE au lycée en contexte marocain.

This contribution returns to the functions and uses of literary genres as advocated in the official curricula for secondary education in Morocco. Some excesses are then highlighted and didactic approaches are proposed. The latter should help to optimize the teaching/learning of FLE at the secondary level in the Moroccan context.

\section{INDEX}

Thèmes : Varia

Mots-clés : fonctions du genre littéraire, enseignement/apprentissage, textes littéraires, usage Keywords : functions of the literary genre, teaching/learning, use, literary texts

\section{AUTEUR}

\section{MINA SADIQUI}

Ecole Normale Supérieure, Université My Imail, Maroc

Habilitée à diriger des recherches en D.L.C

Formatrice de professionnel.le.s de l'éducation

Responsable de la une structure de recherche Education, Formation Et Enseignement/

Apprentissage des Langues (EFEL), Laboratoire RDLLC. Responsable de la filière universitaire d'éducation « Didactique du français »

Parmi ses dernières publications : collectif, Le français au Maroc au XXIe siècle - Vers de nouveaux enjeux ?, L'Harmattan (2019)

m.sadiqui[at]ens.umi.ac.ma 